\title{
Photonic Band Gap Corrugated Slow Wave Structure for THz Sheet-Beam Vacuum Electron Devices
}

\author{
Rosa Letizia ${ }^{1,2}$, Mauro Mineo ${ }^{3}$ and Claudio Paoloni ${ }^{1}$ \\ ${ }^{1}$ Engineering Department, Lancaster University \\ Lancaster, UK, LA1 4YW \\ ${ }^{2}$ Cockcroft Institute, Daresbury, UK, WA4 4AD \\ ${ }^{3}$ E2V Technologies \\ r.letizia@lancaster.ac.uk
}

\begin{abstract}
The use of photonic band gap (PBG) technology is investigated to alleviate some of the typical issues of vacuum electron devices at terahertz and is shown as particularly suitable for the use of large sheet beams. A full interaction structure including the slow wave structure and the coupler based on a tapered PBG corrugated waveguide is proposed for sheet beam backward wave oscillators (BWO). The case of a 346 GHz BWO is considered.
\end{abstract}

Keywords: Photonic band gap; backward wave oscillator; Terahertz; sheet beam.

\section{Introduction}

Backward wave oscillators (BWOs) are considered among the most promising device to provide power in the $\mathrm{THz}$ region (100-1000 GHz), [1]. At this frequency range, photonic band gap (PBG) technology has been recently proposed to alleviate some of the challenges deriving from the use of metallic periodic waveguides with the dimensions in the sub-millimeter range, [2-4]. In particular, the use of PBG structures allows to use a rectangular beam tunnel for very wide sheet electron beams which does not require the implementation of impedance mismatching circuits. In addition, compared to conventional interaction structures used in vacuum electron devices (VEDs), the benefits include the openness of the waveguide to ease the vacuum pumping, easy assembly, flexibility and scalability of the design. In particular an air gap between the PBG and the top lid would simplify the fabrication.

Slow wave structures (SWS) based on a corrugated waveguide can interact effectively with wide sheet electron beams (cross sectional ratio greater than 5:1) which can support high level of current for a given cathode loading and have recently emerged as a promising solution for relatively high output power VEDs in the $\mathrm{THz}$ frequency range. In our recent work, we have demonstrated that the metal walls of a conventional corrugated waveguide can be effectively replaced by opportunely designed PBG structures, [2]. In addition, tapered photonic band gap (TPBG) structures still offer an effective confinement of the useful RF signal while maximizing the empty region around the central corrugations. In this paper, the TPBG corrugated waveguide is designed for a BWO at $346 \mathrm{GHz}$. The 346 $\mathrm{GHz}$ is a relevant frequency in plasma diagnostics for nuclear fusion. A suitable and robust coupler is also designed to be embedded in the same TPBG structure.

\section{Tapered PBG Corrugated Waveguide}

A metallic PBG waveguide is designed to operate around $346 \mathrm{GHz}$ with width of the central channel $w=0.71 \mathrm{~mm}$, and height $b=0.36 \mathrm{~mm}$. The $2 \mathrm{D}$ periodic structure is based on a square lattice of copper pillars of period $\mathrm{a}_{\mathrm{p}}=0.3$ $\mathrm{mm}$ and radius $r_{p}=0.06 \mathrm{~mm}$. The band diagram for the PBG structure closed between two metal plates at the distance of $0.36 \mathrm{~mm}$ is shown in Figure 1. From this Figure it can be seen that a complete band gap exists at operation. It has been previously shown that a number of four pillars on each side of the central corrugation is sufficient to provide confinement of the signal within the PBG waveguide. Starting from this PBG structure, a modified design is proposed where the radius of the pillars is increased along the transverse direction towards the edges of the SWS. A schematic of the proposed TPBG corrugated waveguide designed at $346 \mathrm{GHz}$ is shown in Table I. The central corrugation has period of $0.1 \mathrm{~mm}$ and length of $50 \mu \mathrm{m}$. Assuming a beam thickness of about 20 $\mu \mathrm{m}$, these specifications are suitable for sheet beam ratios up to $15: 1$.
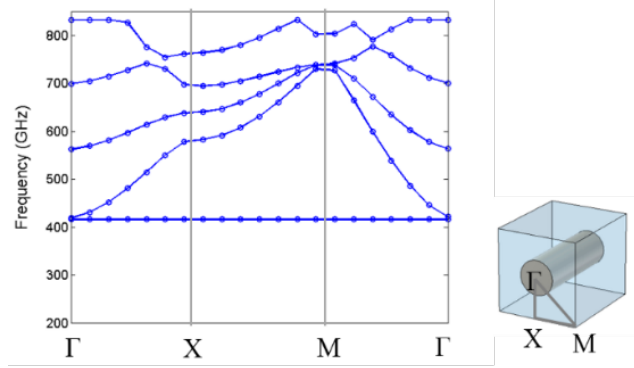

Figure 1. Band diagram for the PBG structure with height of $0.36 \mathrm{~mm}$ sandwiched between metal plates. The unit cell is shown in the inset.

Figure 2 shows the comparison between the dispersion diagram obtained for the TPBG corrugated waveguide, the uniform PBG corrugated waveguide with dimensions as described in Figure 1 and the conventional corrugated waveguide which shows similar behavior. 


\section{Tapered PBG coupler}

A suitable coupler is designed as a 90 degree PBG-bend embedded in the same tapered PBG structure as shown in Figure 3. The performance of the coupler is optimized by tailoring a number of defects $\left(\mathrm{P}_{1}-\mathrm{P}_{2}-\mathrm{P}_{3}\right.$ in Figure 3) to optimize the signal coupling into the output port and obtain a very effective design for the beam tunnel. The detailed geometry of the TPBG coupler for $346 \mathrm{GHz}$ can be found in [3].

Table 1. TPBG corrugated waveguide geometry

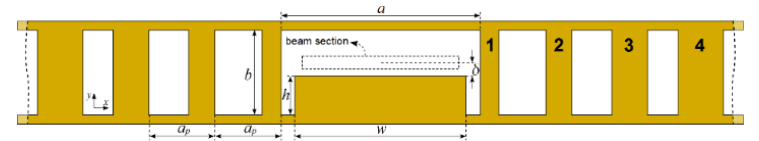

\begin{tabular}{|c|c|c|c|c|}
\hline Pillar & $\mathbf{1}$ & $\mathbf{2}$ & $\mathbf{3}$ & $\mathbf{4}$ \\
\hline $\mathbf{r}_{\mathbf{p}}(\mathbf{m m})$ & 0.04 & 0.05 & 0.07 & 0.08 \\
\hline $\mathbf{r}_{\mathrm{p}} / \mathbf{a}_{\mathbf{p}}$ & 0.13 & 0.17 & 0.23 & 0.27 \\
\hline $\mathbf{a}(\mathbf{m m})$ & \multicolumn{3}{|c|}{0.71} \\
\hline $\mathbf{b}(\mathbf{m m})$ & \multicolumn{3}{|c|}{0.36} \\
\hline $\mathbf{a}_{\mathbf{p}}(\mathbf{m m})$ & \multicolumn{3}{|c|}{0.30} \\
\hline $\mathbf{h}(\mathbf{m m})$ & \multicolumn{3}{|c}{0.16} \\
\hline $\mathbf{w}(\mathbf{m m})$ & \multicolumn{3}{|c}{0.54} \\
\hline
\end{tabular}
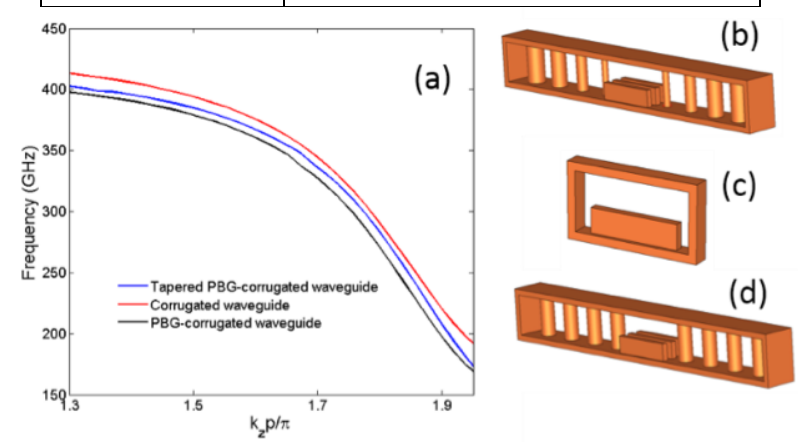

Figure 2. Dispersion curve comparison (a) for the TPBG corrugated waveguide, shown in (b), the conventional corrugated waveguide (c), and the PBG corrugated waveguide $(\mathrm{d})$.

Here, simulation results show that the behavior of the coupler is maintained even in the presence of air gap between the pillars and the top lid. The S-parameters results are shown in Figure 4, where return loss is less than $-30 \mathrm{dBs}$ and transmission into the beam tunnel is lower than $-45 \mathrm{dBs}$ around operation.

\section{Conclusions}

The design for a $\mathrm{BWO}$ at $346 \mathrm{GHz}$ based on a tapered PBG structure has been proposed. With respect to conventional corrugated waveguides for sheet electron beams interaction at the $\mathrm{THz}$ range, tapered $\mathrm{PBG}$ corrugated waveguides offer the advantages of being a flexible, and effective way to alleviate typical problems of $\mathrm{THz}$ tubes such as the low cutoff for sheet beam tunnel and time consuming vacuum pumping of closed structures. The coupler is robust and maintains its performance when an air gap up to $37 \mu \mathrm{m}$ exists between the pillars and the top lid.

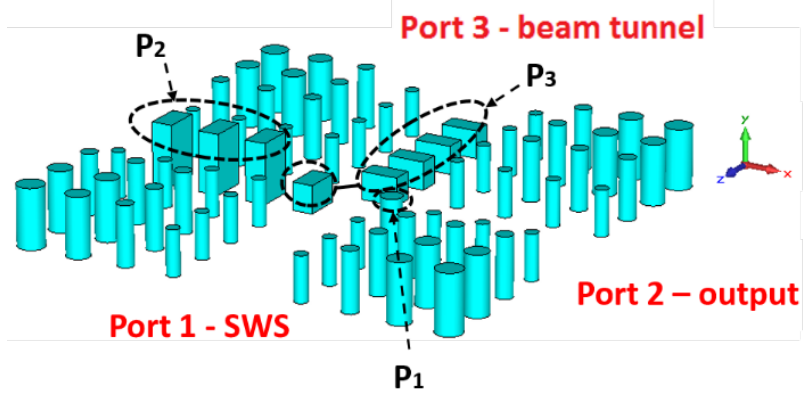

Figure 3. TPBG-coupler schematic. The defects $P_{1}, P_{2}, P_{3}$ are strategically placed to maximize the transmission in Port 1. The height of pillars $P_{3}$ is reduced to $0.16 \mathrm{~mm}$ to allow for the beam to be injected.
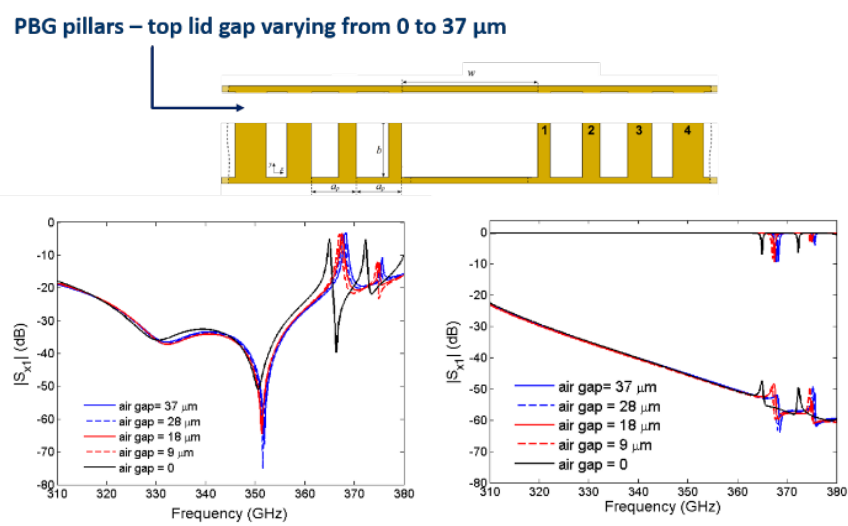

Figure 4. S-parameters for the TPBG-coupler with top lid air gap varying from 0 to $37 \mu \mathrm{m}$

\section{References}

1. Letizia, R., M. Mineo, and C. Paoloni, "Photonic Crystal-structures for $\mathrm{THz}$ Vacuum Electron Devices," Electron Devices, IEEE Transactions on, vol. 62, no. 1, pp. 178-183, Jan. 2015

2. Booske, J. H.,et al. "Vacuum electronic high power terahertz sources," IEEE Trans. Terahertz Sci. Technol., vol. 1, no. 1, pp. 54-75, Aug. 2011

3. Letizia, R., M. Mineo, and C. Paoloni, "Photonic Bandgap Coupler for $346 \mathrm{GHz}$ Sheet-Beam BWO," Proc. of UCMMT, Cardiff, UK, Sept. 2015

4. M. Mineo and C. Paoloni, "Corrugated rectangular waveguide tunable backward wave oscillator for $\mathrm{THz}$ applications", Electron Devices, IEEE Transactions on, vol. 57, pp. 1481-1484, 2010 